\title{
Examining Groundwater and Surface Water Interactions to Determine the Effects of Anthropogenic Nutrient Loading on Streams and Coastal Water Quality
}

Michael R. Mathioudakis, Craig R. Glenn, Daniel E. Dores

University of Hawai'i at Mānoa, Department of Geology and Geophysics

\section{Introduction}

Submarine groundwater discharge (SGD) is an essential source of land-derived nutrients for coastal waters, but excess anthropogenic nutrient loading (e.g. from agriculture or cesspools) can significantly degrade coastal ecosystems.

Groundwater is insulated from the sun, so temperature gradients exist between discharging groundwater and receiving surface water bodies. These temperature differences are easily detectable by thermal infrared (TIR) imaging.

Recent studies have utilized aircraft and/or ground-based TIR to survey coastal areas, estuaries, and rivers/streams for groundwater inputs. However, these methods have significan limitations, as Aircraft-TIR can be limited by cloud cover, and ground-based TIR is impractical for surveying large areas.

UAV-TIR is ideal for surveying SGD on a small scale because it allows unprecedented data collection flexibility and highresolution below-cloud TIR imaging.

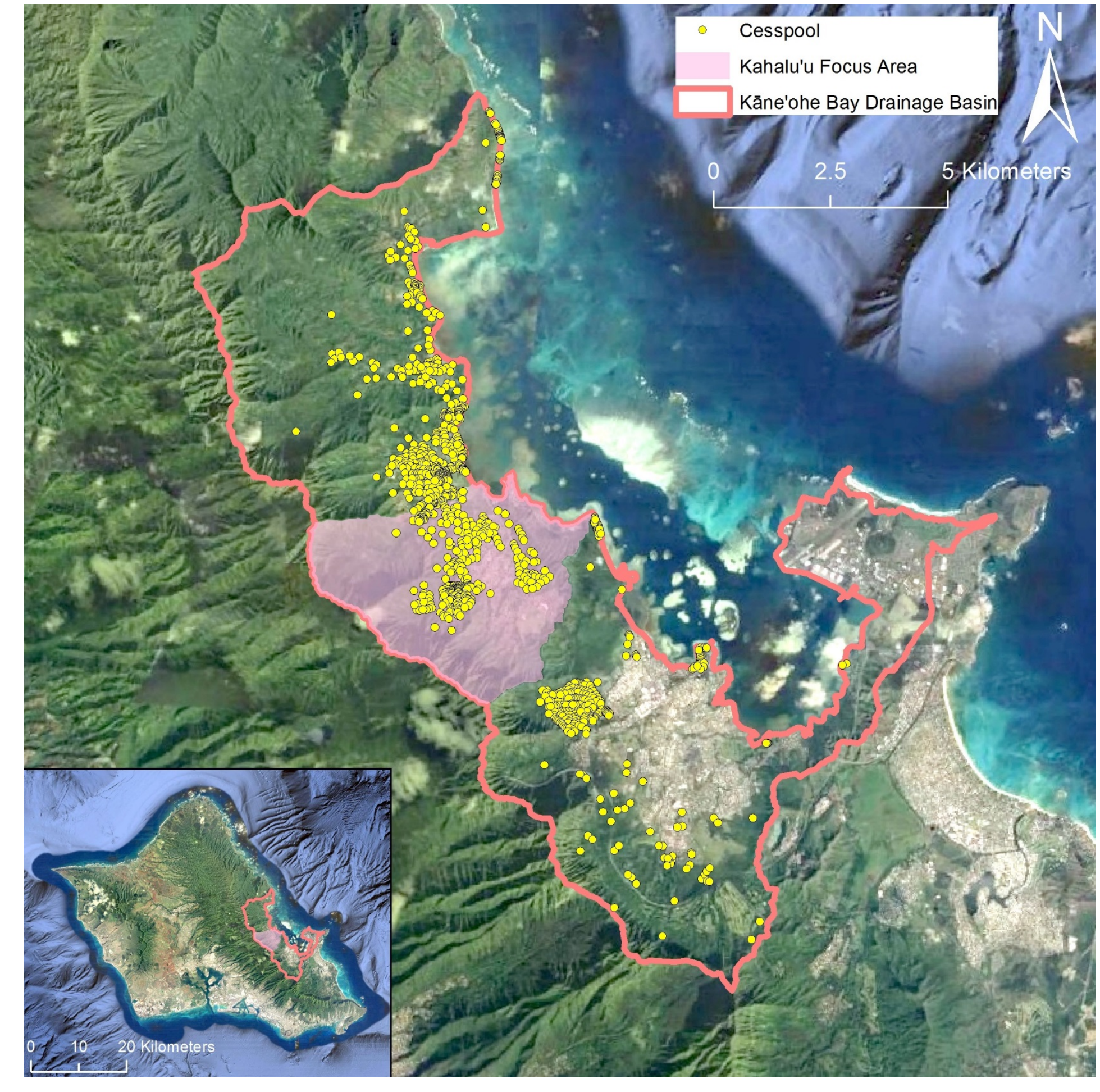

Figure 1: Map of the Kāne'ohe Bay drainage
basin showing the distribution of cesspools, a potential source of anthropogenic nutrients in

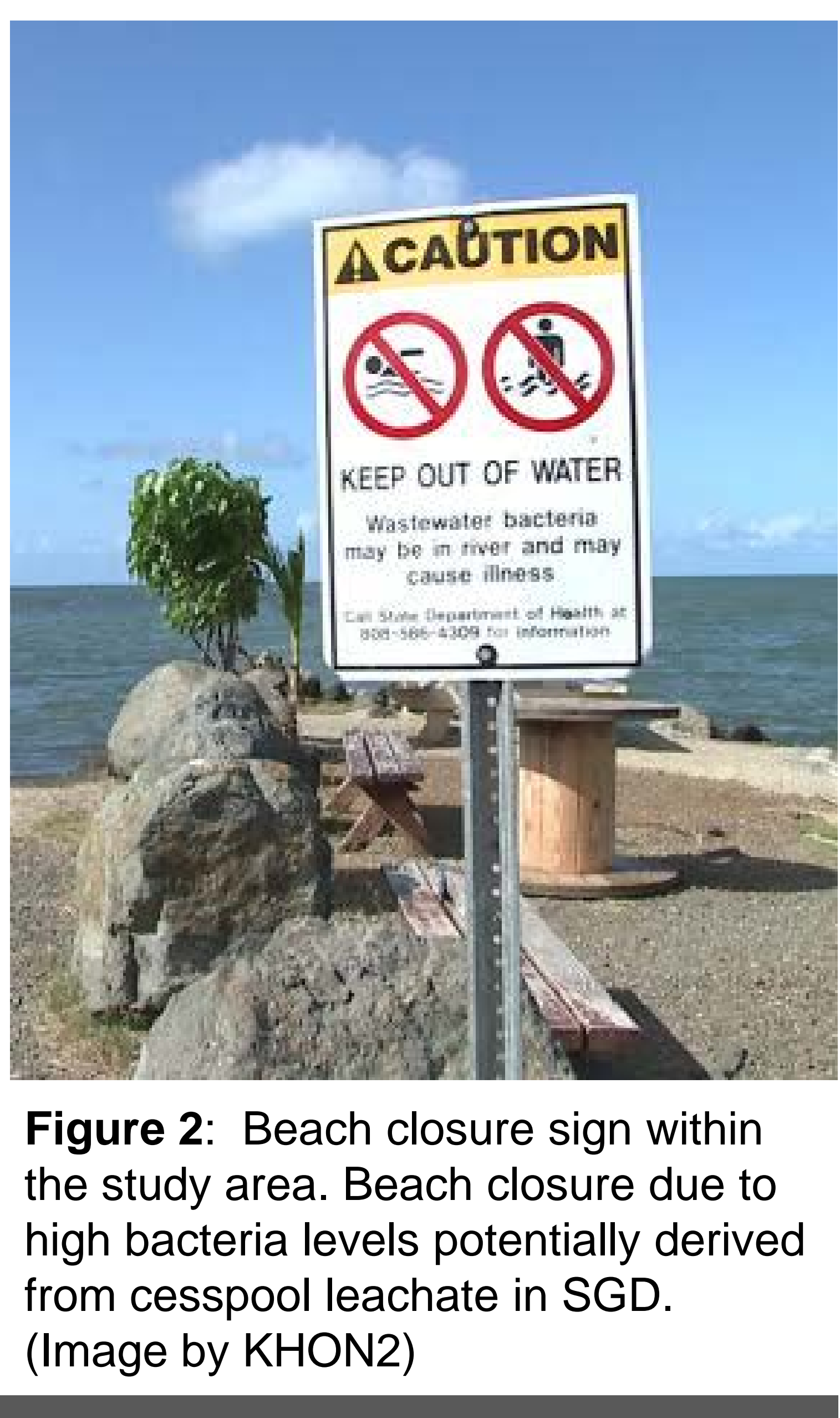

Goals
Identify locations of submarine groundwater discharge in Central Kāne'ohe Bay, based on thermal contrast between groundwater and surface water.

Previous studies (Lee et al., 2016; Kennedy et al., 2017) utilized UAV-TIR as a follow-up to Aircraft-TIR studies. The goal of this study is to demonstrate effective independent use of UAVTIR for locating and mapping SGD.

\section{Methods}

Equipment

UAV Platform

Power

Autopilot

TIR Camera

Focal Length

Pixel Resolution

Temperature Resolution

Image Capture

3DR X8+ Octocopter
$14.8 \mathrm{~V}$ Lithium Polymer batteries
Pixhawk
FLIR Tau 2640
$13 \mathrm{~mm}$
$0.2 \mathrm{~m}$
$0.04^{\circ} \mathrm{C}$
TeAx Thermal Capture

\section{Data Collection}

10-12 minute flights, 120 meter altitude

Flights conducted during low tide to maximize hydraulic gradients, and late in the day to maximize thermal gradients

In-situ thermistors deployed during flights to ground-truth TIR data Thermal imagery collected at a rate of $9 \mathrm{~Hz}$ (9 frames per second)

Spatial data attached to each image via on-board GPS unit

Data relayed to ground station monitor for real-time analysis

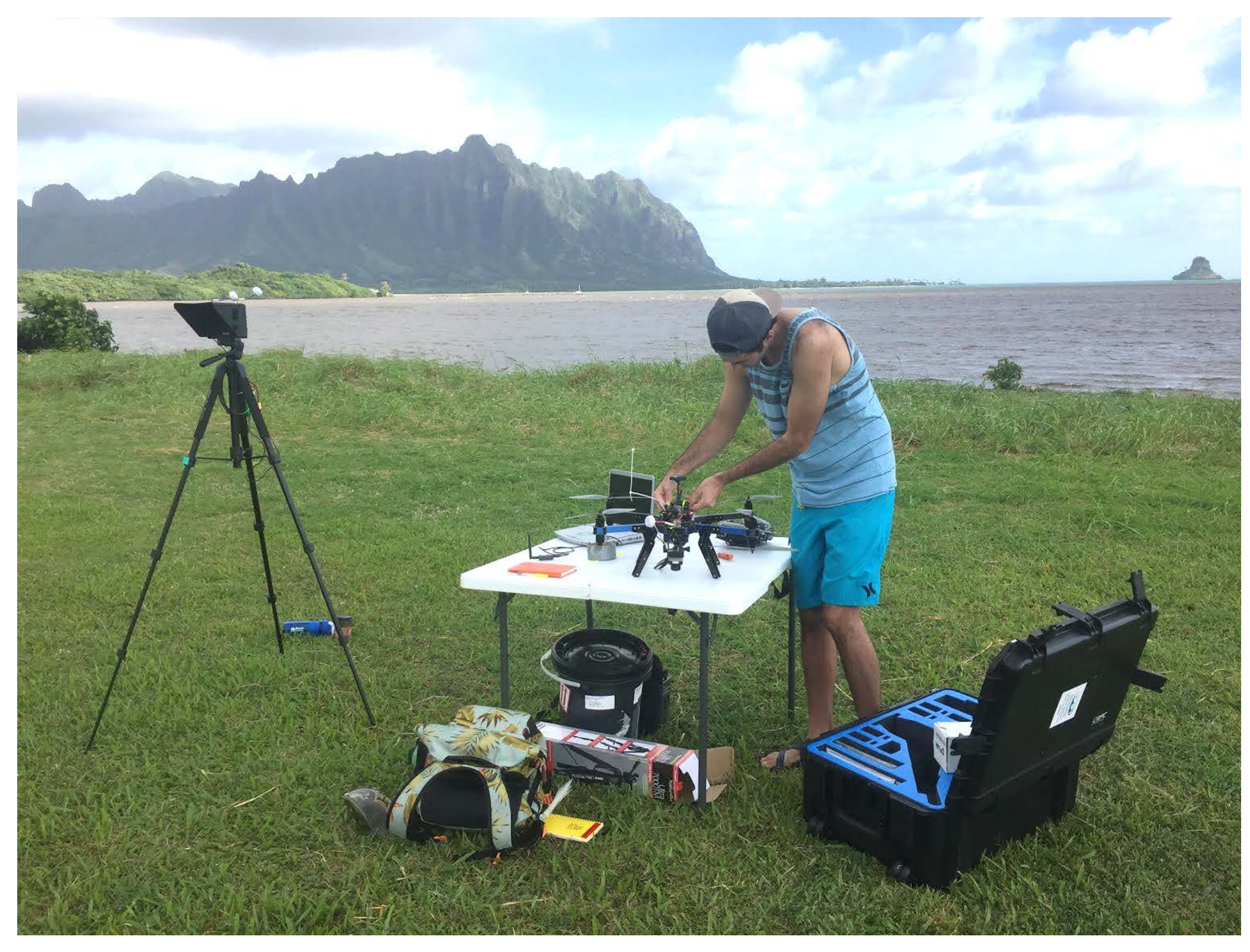

Figure 3: UAV-TIR field operations in Kāne'ohe.

Post-Processing

raw thermal files converted to grayscale JPEGs

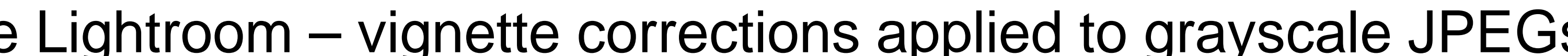

Microsoft ICE - image mosaicking with corrected grayscale JPEGs

ArcGIS - grayscale mosaics converted to temperature rasters

\section{Paired Methods}

UAV-TIR provides the potential for locating, mapping, and analyzing the areal extent of SGD. Pairing UAV-TIR with other methods (e.g. simultaneous radionuclide tracer measurements, simultaneous vertical temperature profiling follow-up geochemical studies) can provide furter qualitive he method to predict and/or confirm SGD locations.

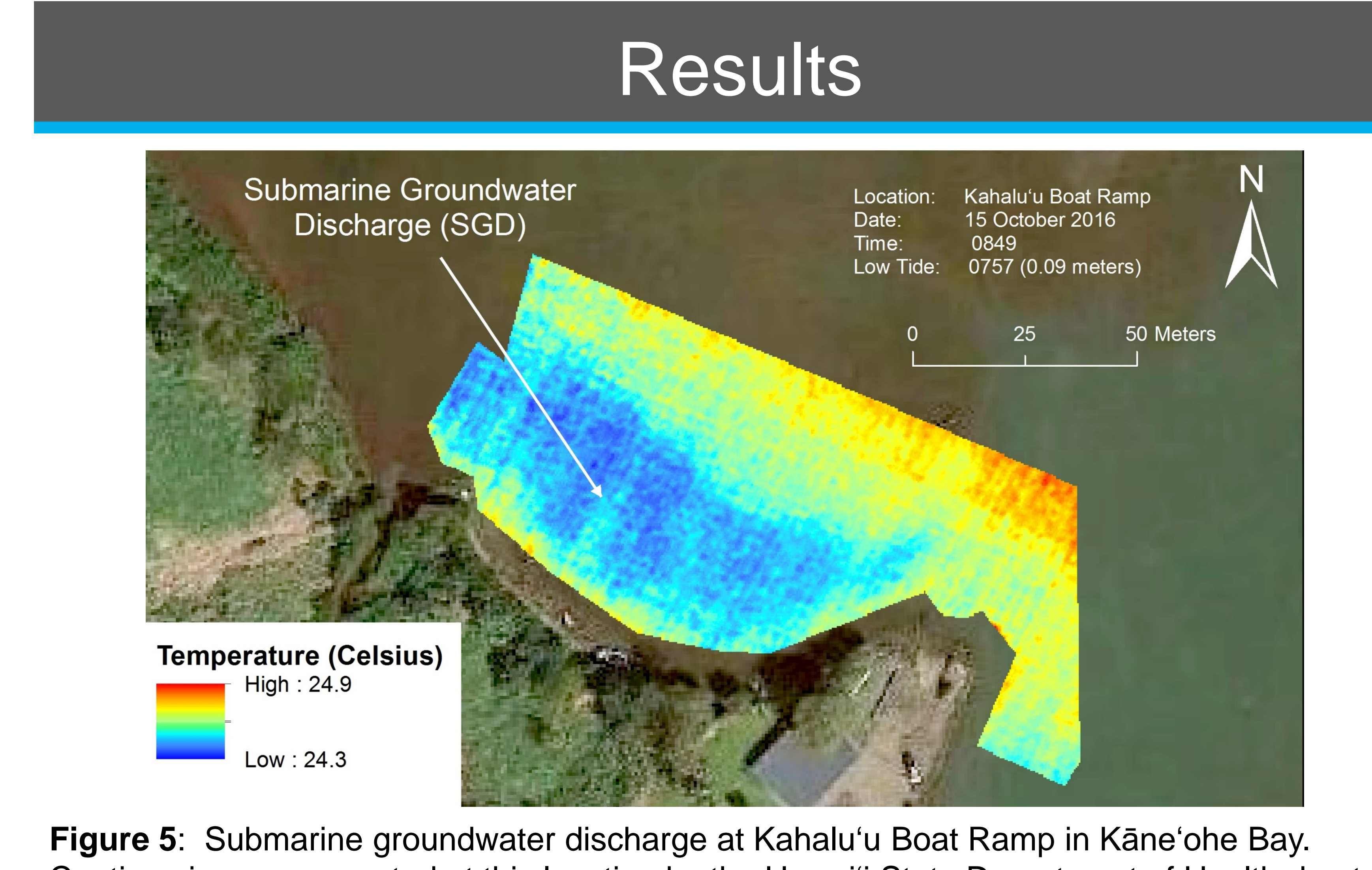

Figure 5: Submarine groundwater discharge at Kahalu'u Boat Ramp in Kāne'ohe Bay.
Caution signs were posted at this location by the Hawaiti State Department of Health due to Caution signs were posted at this location by the Hawali

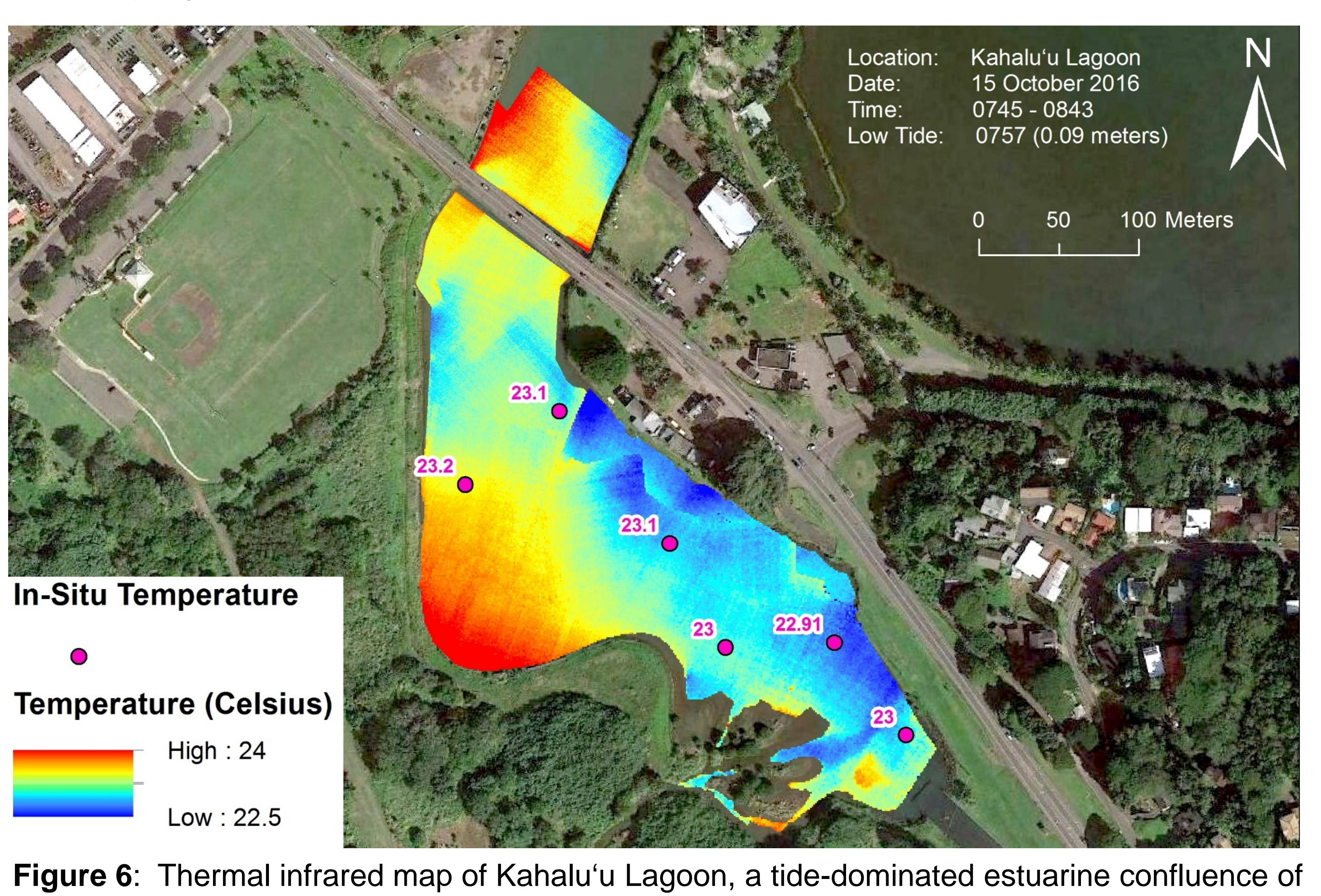
Figure 6: Thermal infrared map of Kahalu'u Lagoon, a tide-do
three streams (Kahalu'u, Waihe'e, Ahuimanu) in the study area.

\section{Conclusions / Future Work}

The use of UAV-TIR in Kāne'ohe Bay has allowed us to easily locate submarine groundwater discharge, identify the areal extent of SGD, distinguish between point-source and diffuse discharge, and quantify temperature gradients. UAV-TIR is a flexible and cost-effective tool for mapping and monitoring SGD, independent of Aerial-TIR surveys.

\section{Future Work}

Map the entire Central Kāne'ohe Bay coastline

Follow-up geochemical studies to characterize SGD

Apply these methods to detect stream baseflow

Acknowledgments

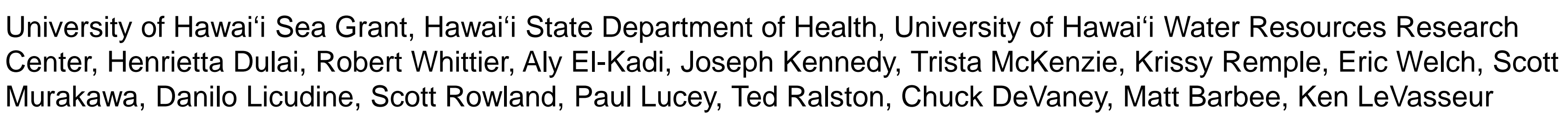

